\title{
A Access Traffic Sign Detection Tracking and Recognition System for Video Frames using KNN Algorithms
}

\author{
D. Siva Reddy, Christeena Joseph
}

\begin{abstract}
Video-based traffic sign detectiontracking and recognition plays an important role in driving support system as well as in intelligent autonomous vehicles. This framework includes three parts like traffic sign detecting, target tracking and sign recognition. This paper contributes different methods for detecting and recognizing of traffic sign, so that drivers can be easily identify the type of signal in road sides. In this approach different methods like color segmentation and scale based intraframe fusion technics is proposed which includes the spatial-temperol constraints in videos, by fusing the different casings that have a place with an equivalent physical sign along to induce higher exactness.
\end{abstract}

Keywords: Traffic sign detection, Tracking, Recognition, Kalman filter,

\section{INTRODUCTION}

Nowadays the vehicles are becoming a primary need of transport within the twenty first century and their range has been increasing step by step from the time they were madeup. At the current scenario, the urban streets in the created and the creating nations are loaded with vehicles and every year 1.5 million peoples were killed in roads across world and 20 to 30 millions of people were injured.To avoid this problem a right solution is implemented for drivers to take protected measurements while travelling. As road signs square measure the vital parameter for the traffic foundation or infrastructure that plays a significant role in control the vehicles. In the proposed method, a video-based traffic sign discovery, following, and acknowledgment is implemented, where the vehicles can recognize the objects and displays the information to the drivers. Traffic signs are used to provide the guidance or information to the drivers.Intelligent transport system(ITS) makes the different traffic clients to be better educated and makes them safe from the accidents. Many techniques have been proposed in intelligent transport system from the past few years, among them programmed traffic sign discovery and acknowledgment is a significant segmentthat informs the drivers in dangerous situation and provides the navigation to the transport vehicles to make the driving safely and more efficiently. Automatic traffic sign detection is an essential task for the traffic regulation and warning the drivers.Traffic sign detection has many uses furthermore, can be connected to numerous applications, for

\footnotetext{
Revised Manuscript Received on September 10, 2019.

D.Siva Reddy, Saveetha School of Engineering Saveetha Institute of Medical and Technical Sciences, Chennai, Tamilnadu, India.

(Email: dornalasivareddy@gmail.com)

Christeena Joseph, Saveetha School of Engineering Saveetha Institute of Medical and Technical Sciences, Chennai, Tamilnadu, India.

(Email: christeena003@gmail.com)
}

example, self-governing driving propelled driving help framework and in portable mapping.

\section{LITERATURE REVIEW}

2.1 Escalerate al, In the paper, "Road Traffic Sign Detection and Classification" proposeda strategy for the impression of traffic signs by picture examination. Thiswork has three primary jobs a)Road discovery b) hindrance location, and the c) sign acknowledgment. Sign detection is carried out by fractal texture segmentation and neural networkswere images are taken as the input in the form of $\mathrm{N} x \mathrm{~N}$ pixel. This work is presented as a two steps the first one is used to localize thesign in the picture contingent on the size and state of the picture shading. The second is used to recognize the sign through a neural network. In this paper ,performance of up to $99 \%$ correct recognition rate with different signs and conditions.

2.2.Khaled M. Almustafa, in the paper "Circular Traffic Signs Recognition Using The Number Of Peaks Algorithms" focuses on the circular traffic signs for detecting the objects like signs that are presented in the circular shape. This system uses the no. of peaks algorithm that differentiate 51 types of circular shapestreet signs in a quick and solid way. When a particular traffic sign is detected, the algorithm used in this system will draws three flat lines and three vertical lines over the round picturefor calculating the no. of peaks in the scanned image. The resulting no. of peaks algorithm differentiate the 51 circular shape traffic signs for obtaining the detected image in a 8 milliseconds by using the decision-tree-like search algorithm. This system is accurate with $100 \%$ detection rate and can only detect the circular shape objects.

2.3Walietal in the paper"An Automatic Traffic Sign Detection and Recognition System Based on Colour Segmentation, Shape Matching, and SVM" developed a traffic sign identification and recognition(TSDR) framework which contains more dataset of a traffic sign. In this paper, the creators had created three working stages: picture preprocessing, identification and acknowledgment. In the picture preparing stage, the pictures are caught by the onboard camera under various climate conditions and picture pre-handling was finished utilizing the RGB shading division. It likewise utilizes a help vector machine (SVM) for acknowledgment reason with the sacked piece. In this paper the members accomplished with exactness execution of $95.71 \%$, false rate 0.009 , and the handling time is $0.43 \mathrm{~s}$. 
2.4 Kuangetal in the proposed work "Real-Time Detection and Recognition of Road Traffic Signs using MSER and Random Forests" implementeda traffic sign location and acknowledgment technique on picture upgraded maximally steady extremal regions(MSER)and arbitrary woodlands. The force of brightening is amended utilizing a dark world calculation, to improve the precision region of interest(ROI) extraction the CLAHEwas utilized to upgrade picture and it additionally used to improve the complexity. The exploratory outcomes acquired by the creators demonstrate that the proposed new technique has great power to the brightening conditions and achieves well exhibitions under two conditions as the ordinary and feeble light.

2.5 Leonardo Bruno in the proposed work "Improved Traffic Signal Detection and Classification via Image Processing Algorithms" implemented a calculation for identifying, arranging and acknowledgment of the street signs was introduced. They built up a self-governing technique which is utilized for both street stock and after that the upkeep of the current street arrange. This methodology causes the drivers to perceive contrasts in street signs concerning their position and size. The outcomes gotten by them demonstrates that over $98 \%$ of the signs are effectively recognized through picture examination, however, in urban zones, the proposed calculation was not ready to identify more than one sign when the billboards are harmed and uncolored.

\section{Software Description}

For the most part, the term computerized alludes to the handling of a two-dimensional picture by an advanced PC. In an expansive running setting, it demonstrates the computerized preparing of any 2-dimensional data. The propelled ranges diagrammatic by a predetermined number of bits. The picture is given inside the kind of a photograph or an X-shaft is then at first digitized and kept as a structure of matched digits in PC hardware. This digitized picture will by then be taken care of and appeared on a high-objectives TV screen. To demonstrate the image, it should be secured in fast access bolster memory, that restores the screen at a rate of twenty-five edges for each second to give an apparently incessant introduction.

\section{IMAGE PROCESSOR:}

In computerized it will do the elements of the accompanying: picture securing, pre-preparing, capacity, division, acknowledgment, portrayal, understanding lastly record or show theconsequent picture. The underneath square outline givesthe natural grouping required during this framework.

As appeared in the above square graph, the essential advance is a picture securing by an imaging sensor identified with a digitizer to digitize the image. The accompanying stageis the pre-handling step where the pictures are improved being given as a contribution to the followingstrategies. Pre-handling fundamentally overseescomputerized pictures for upgrading, expelling clamour, detaching areas, and so on. The next process is Segmentation, it divides a picture or image into its computerized picture is alluded to as, a variety of genuine or

constituent parts or thingsand afterward the yield will be for the most part, as unrefined pixel data, that includes either the farthest point region or the pixels inside the region themselves.

\section{Pre-processing :}

Digital image processing makes the processing of any kind of image into digital form. Recent cameras may take the image directlyinto digitalformatand these images are generally the images are originated in the form of optical format. This images are captured bythe video cameras and stored in the form of digitalized formats. The digitalization process consists of two technics, sampling and then quantization. By then, these photos are taken care ofby the crucial procedures contains five modules, which is used to synthesize the digital formats. The image processing consist of various technics:Picture improvement, rebuilding, investigation, pressure and picture union.

\section{METHODOLOGY}

In all the present ways the pictures square measure processed for the traffic sign detection. during this planned technique, video frame is employed and traffic signs were extracted from the moving frames. The summary of this technique is shown in fig 2.The planned work uses 5 stages, AN input video frame that is taken from the moving vehicle, then the off-line find or is employed to detect the traffic sign and also the detected traffic signs square measure bornagain into the motion model. once process the following frames, the movement model is refreshed and acquainted with foreseeing the pursuit results. interim the on-line test collection algorithmic program can look at the believability of the normal outcomes by the movement model. Whenever guaranteed, the pursuit results at that point will be utilized as on-line tests to mentor an on-line indicator gradually. If not guaranteed, the on-line discoverer is utilized to distinguish provincially and furthermore the identified outcomes were an acquainted with update inside the movement model. a definitive discovery and pursue results square measure the yield of the movement model once guaranteed or the on-line location results once not guaranteed. other than the location and pursue arrange, the half-track results square measure ordered and joined along to actuate the last acknowledgment yield steadily.

\section{Block Diagram:}

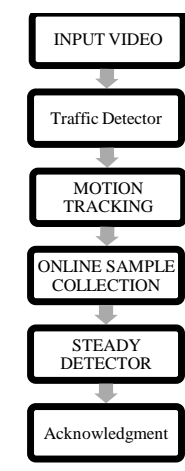

FIG 2.0 Proposed work 


\subsection{Classification of Traffic Signs:}

There are a few several traffic signs accessible covering a wide assortment of traffic circumstances. These traffic signs are classified into three main categories.

Regularity Signs: These signs are accustomed indicate the driver force to adapt the signs for the safety of alternative road users.

These signs are additionally alluded to as required signs or compulsory signs since it is obligatory that the drivers ought to comply with these sort of signs. These signs are basically suggested for the security of other road customers. These signs have regularly dull legend on a white establishment. They are indirect perfectly healthy with the red edges.

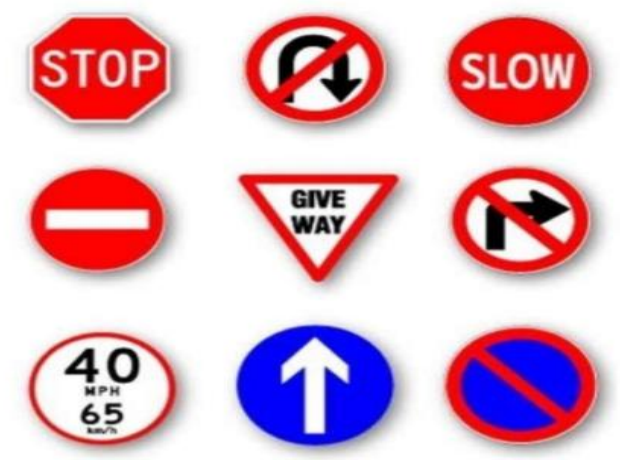

FIG 2.1 Regularity Signs [11]

Warning Signs: These signs are used for the prosperity of oneself who is driving and urging the drivers to conform to these signs.

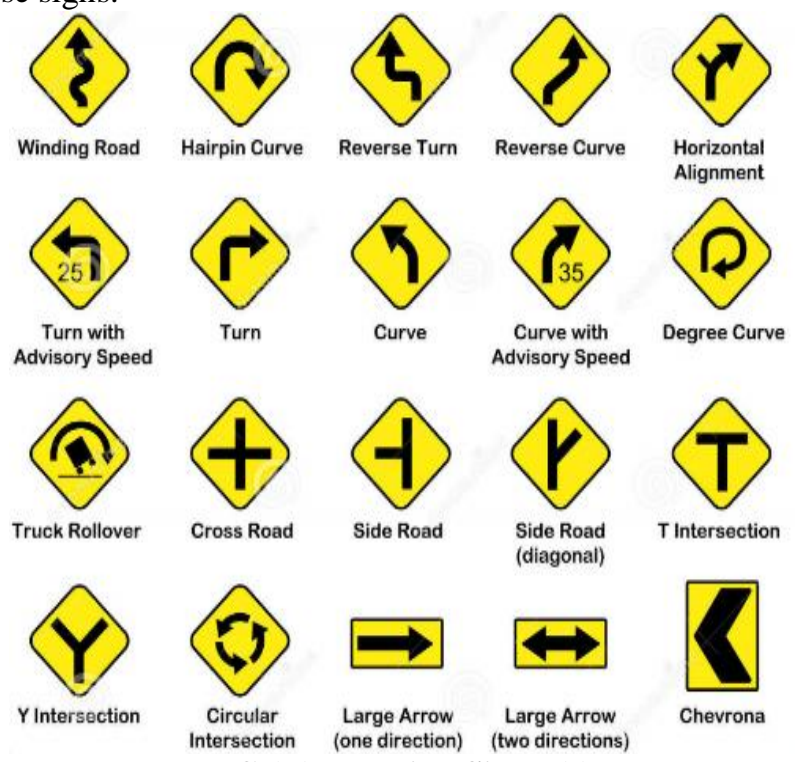

FIG 2.2 Warning Signs[11]

Warning signs are referred to as cautionary signs that provide data to the motive force concerning the road condition. These signs indicates the drivers for own safety. They involvefurther carefulness from the piece of drivers. The shading shows utilized for this kind of signs is that the legend will be dark in shading.

\section{Informative signs:}

These signs give information to the drivers where the objects are placed. These signs are generally used to follow normal peoples to identify the route and distance ahead.

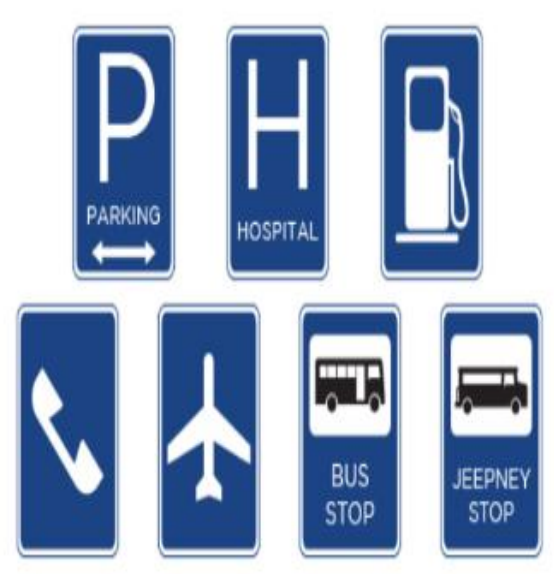

FIG 2.3 Informative signs[11]

\subsection{Traffic Sign detection}

Traffic sign might be distinguished with the help of 2 finders one is disconnected locator and in this way, the option is the online identifier. For a substitution input video outline, Associate in Nursing disconnected watch or are connected to identify the hopeful traffic signs. The identifier is prepared in advance and remains unaltered all through the full technique. This may ensure a steady character of the full system while the accommodating half is handled by the consequent online thought. By instinct, shading is a fundamental signal for article recognition. On the off chance that the disconnected locator does not refresh information, that was kept to the movement model then the web indicator was utilized to change the information to the movement. In fig 2.5 the demonstrates the location of a traffic sign.

\section{Algorithm analysis}

Gauge street sign after depends on the Kalman-Bucy sifting method ordinarily embraced in a few past examination on traffic sign recognition(TSR). this framework makes an endeavour to display exclusively the unadulterated science of the objective over the time and it included 2 sections: Kalman Filter (KF) for position and scale desire, still as a zone search district estimation, and a Particle Filter (PF) wont to address the anisotropic scaling of the perceived sign. Since the Kalman channel having a general drawback of surveying the state $x \in R^{\wedge} n$ of the direct procedure:

$x_{t+1}=\mathrm{A} x_{t}+\mathrm{B} u_{t}+w_{t}$,

Given the observations $\mathrm{z} \in R^{m}$ :

$z_{t}=\mathrm{H} x_{t}+v_{t}$.

Where $\mathrm{H}$ is that the deliberate lattice relating the concealed state variable $\mathrm{x}$ with the vector of perceptions $\mathrm{z}$. Since Kalman filter is failing in some things like, once the sign boards square measure get broken or crying. $\mathrm{K}$ nearest neighbour(KNN) rule is employed to work out the pattern recognition and to live the space a scale primarily based frame fusion technic is bestowed. supported Gauge street sign after depends on the Kalman-Bucy sifting method ordinarily embraced in a few past examination on traffic 
sign recognition(TSR). this framework makes an endeavour to display exclusively the unadulterated science of the objective over the time and it included 2 sections: Kalman Filter (KF) for position and scale desire, still as a zone search area estimation, and a Particle Filter (PF) wont to address the anisotropic scaling of the perceived sign. Since the Kalman channel having a general drawback of evaluating the state

\subsection{Target Tracking}

Following street signs from a vehicle-mounted camera isincredibly tough for many reasons, as a result of the vehicles might bear non-uniform motion, as an example it's going to speedily accelerate, brake, or modification the driving direction whereas reordering another vehicle. This development is tough to model.Be that as it may, in unfriendly brightening, every property can't be abused absolutely on the grounds that the qualification around the sign's limit and its pictogram images are way less sharp and along these lines, the unremarkably particular hues appear to be pale. moreover, shadows and episode lightweight reflections, which as often as possible happen in rush hour gridlock scenes, may affect the consistency of the track. To maintain a strategic distance from this trouble one in everything about for the most part utilized methodology is transient street sign traffic depends on Kalman filtering(KF). this is frequently acquainted with foreseeing the position and size of the objective inside the casings of the in information video and consequently proportional back the district. From fig 2.6 shows however the motion is pursuit victimisationthe Kalman filter and its motion was foretold at each frames within the input video.

\subsection{Traffic Sign Recognition}

In current circumstance traffic sign acknowledgment is significant for independent vehicles and for manual drivers to stay away from the mishaps. Road sign recognition involves 2 essential tasks: feature extraction and classification. it's terribly tough to spot the actual sign and it used the KNN formula, wherever it'll collect all info from the item and supported the colorsegmentationit acknowledges.K nearest neighbours (KNN) may be a straightforward formula that stores all the on the market traffic signs and classify new signs supported the space the vehicle is traveling. fig 2.7 displays the sign recognition and it additionally identifies kind of sign . during this a scale based mostly intra-fusion was enforced, this technic combine the aftereffects of different edges that have a place with an indistinguishable physical sign along to initiate an improved precision.

KNN formula is one amongst the best classification formula and it's one amongst the foremost used learning algorithms. KNN perhaps a non-parametric, languid learningformula. Its purpose is to use a info at intervals that the information purposes are isolated into numerous classes to foresee the arrangement of a substitution test point.

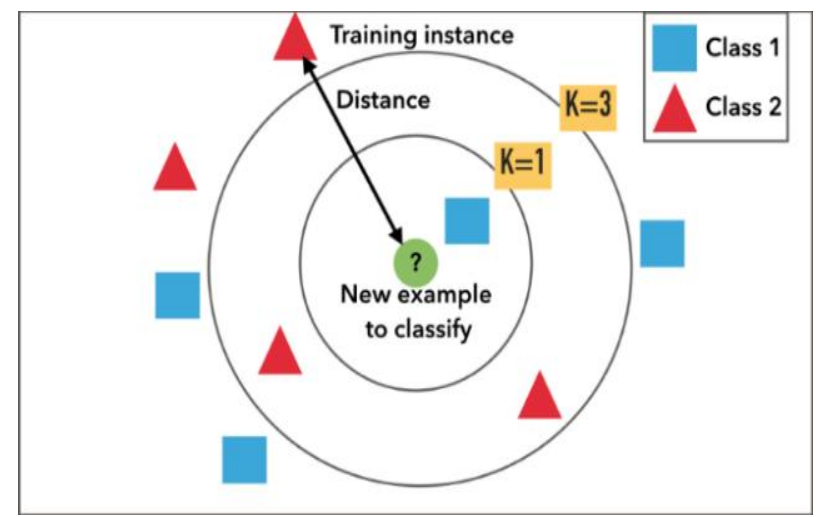

FIG 2.4 example of KNN algorithm classification

\subsection{Algorithm for overall detection and tracking.}

Instate: for $i=1$ to $m$ do

Peruse test $s i=(x i, y i)$

update gradual svm classifier

Mon end for spare model Mon While(fk):

disconnected identification Moff( $f k): s m k=(x m k$, $y m k, w m k, h m k)$

Kalman Filter Process:

Prediction phase:

$s p k=(x p k, y p k, w p k, h p k)=(F X s e(k-1)+B u k) P k \mid k-1=$ $F P k-1 \mid k-1 F T+Q$

On-line Learning: Call Algorithml with info spk

on the off chance that L positive $(s p k)<\tau$

On-line Detection:Call Algorithm2 get smax

Last consequence of casing $k$ :

sfk = smax

Update phase: $K k=P k \mid k-1 H T(H P k \mid k-1 H T+R k)-1$

sek $=\operatorname{smax}+K k(\operatorname{smax}-H) s p k$

else Update phase: Kk $=P k \mid k-1 H T(H P k \mid k-1 H T+$

$R k)-1$ sek $=s p k+K k(s m k-H s p k)$

Final result of frame $k: s f k=$ sek

end if

end While

\section{IV.EXPERIMENTAL RESULTS}

In this section associate degree experimental analysis of comparable algorithms, K nearest neighbour(KNN), Kalman filter and thereforethe portion relapse trees are offered inside the setting of traffic hint acknowledgment. In driving help system, the most objective isn't to quantify similarity between the signs, however to display the proper full implications to the pictograms of signs caughtby camera that is mounted on the vehicle. Algorithms square measure wont to differentiate the signs and square measure place into an order in TSR. In particular, the closest neighbour classifier is built upon the similitudeof live learned victimisation every algorithmic program furthermore, is tried on the static street signpictures and additionally on live traffic video. within the finish of this section, the performance of the 
projected framework and therefore the main elements was analysed.

\subsection{Dataset and setup}

To assess the offered traffic sign acknowledgment approach, an image likeness work was found out from the sets of pictures trimmed from the important life video sequences. For video primarily based traffic sign recognition system, all the information is taken from the moving vehicle. These vehicles are having a mono camera placed before of it and captures all the knowledge through the net detector and stores it in an exceedingly dataset. every sign on this dataset are annotated at 4-5 times at totally extraordinary separation from the vehicle and around 600 edited sign pictures are used for the detector. The traffic live video taken from the camera is hold on in an exceedingly info, and this pictures from the video are regenerate to shut in the $64 \times 64$ pixels(only signs within the image) and $640 \times 640$ pixels.

\subsection{Detection Performance}

The detection performance can be evaluated by using the three measures, precision-recall is adoptedfor various phases of the framework pipeline. For the tracking and on-line detection stage, normalised non-overlapping area is used to measure the localization accuracy. Finally classification rate is used,to use the traffic sign characterization arrange. The exactness review is the parametric bend that speaks to the exchange off among precision and recall. For parallel order issue in example acknowledgment and data recovery, exactness is characterized as the portion of recovered occurrences that are pertinent, while the review has alluded to the part of applicable occasions that are recovered. The exactness review can be detailed as,

$$
\begin{aligned}
\text { Precision } & =\frac{T P}{T P+F P}, \\
\text { Recall } & =\frac{T P}{T P+F N} .
\end{aligned}
$$

To get better accuracy, traffic sign location is significant for the consequent characterization to organize. The image consist of four dimension vectors ( $\mathrm{x}, \mathrm{y}$, width, height) and a rectangular window to employthe separation metric which measures a standardized non-covering region between the two windows. This distance measurement can be formulated as

$$
\operatorname{Distance}\left(d_{x}, d_{x y}\right)=1-\frac{\operatorname{area}\left(d_{x}, d_{x y}\right)}{\max \left(\operatorname{area}\left(d_{x}\right), \operatorname{area}\left(d_{x y}\right)\right)},
$$

For evaluating the classification result,the right grouping rate is utilized to quantify the characterization exactness of the followed traffic signs. The precision-recall comparison curve is shown in fig 2.5, illustrates for three different detection methods.

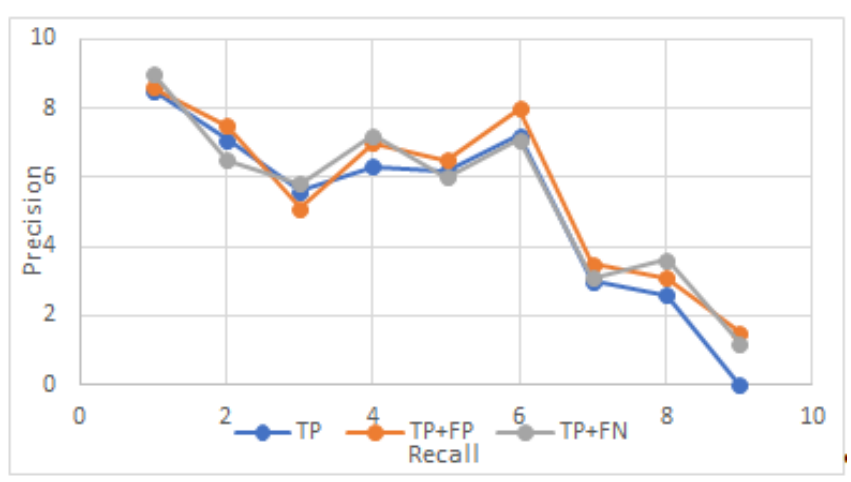

FIG 2.5 comparison between three detection methods.

To distinguish the influence of the various edges on the classification rates, the trials were repetitive for variable estimations of those limits. Results were contrasted and the presentation of the classifier created basically dependent on:

1) the thorough image comparison and

2) picture examination as to the sign portrayals got for the best setting.Below table one. 0 displays the various individual traffic sign recognition results.

Table 1.0

\begin{tabular}{|l|l|l|l|l|l|}
\hline Thresholds $\left(t_{d}\right)$ & & & & & Overall(151) \\
\hline 1.0 & $95.7 \%$ & $85.3 \%$ & $94.2 \%$ & $84.8 \%$ & $90.0 \%$ \\
\hline 2.0 & $95.7 \%$ & $90.1 \%$ & $96.6 \%$ & $93.1 \%$ & $93.8 \%$ \\
\hline 5.0 & $84.5 \%$ & $80.8 \%$ & $97.3 \%$ & $93.1 \%$ & $88.9 \%$ \\
\hline All regions & $82.3 \%$ & $75.7 \%$ & $92.2 \%$ & $82.6 \%$ & $83.2 \%$ \\
\hline Best & $95.7 \%$ & $90.1 \%$ & $97.3 \%$ & $93.1 \%$ & $94.1 \%$ \\
\hline
\end{tabular}

$\mathrm{BS}(16)-16$ blue square traffic boards, YT(45) - 45 yellow color traffic boards

$\mathrm{BC}(25)-25$ blue color traffic signs, $\mathrm{RC}(65)-65$ red color traffic signs.

As seen within the table 1.0, the obtained classification error rate doesn't exceed seven-membered, creating the tactic cherish the most effective condition of craftsmanship approaches. when this classification rate is expanded by the location rate got for a comparative video material inside the analysis depict, almost the vast majority of signs are appropriately distinguished and perceived. The quantities of classes in each classification: red circles (RC), blue circles (BC), yellow triangles (YT), and blue squares (BS) are given in enclosures inside the section headers. The classification rates are resolved from exclusively these signs that were appropriately recognized. the offer shows the location execution of each shading.

\subsection{Results}

Finally, the traffic sign recognition (TSR) performance on dataset was evaluated and enforced the planned colour model based mostly detection technique and uses the tensor flow code technique for profound learning identification and arrangement procedure. a definitive recognition and arrangement results territory unit appeared in the beneath 
figures. Some traffic sings with the minimal size or mutilation is recognized while the standard techniques may neglect to perform.

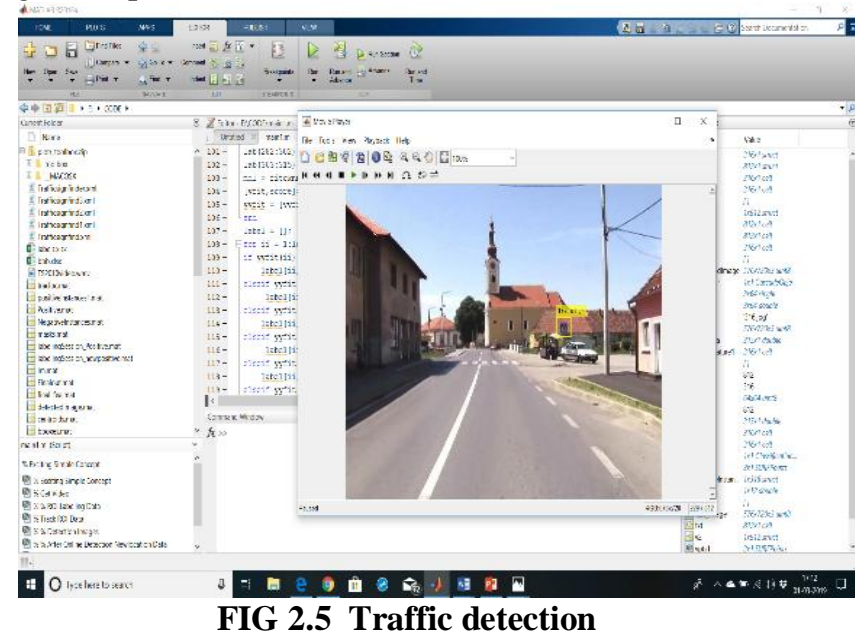

FIG 2.5 Traffic detection
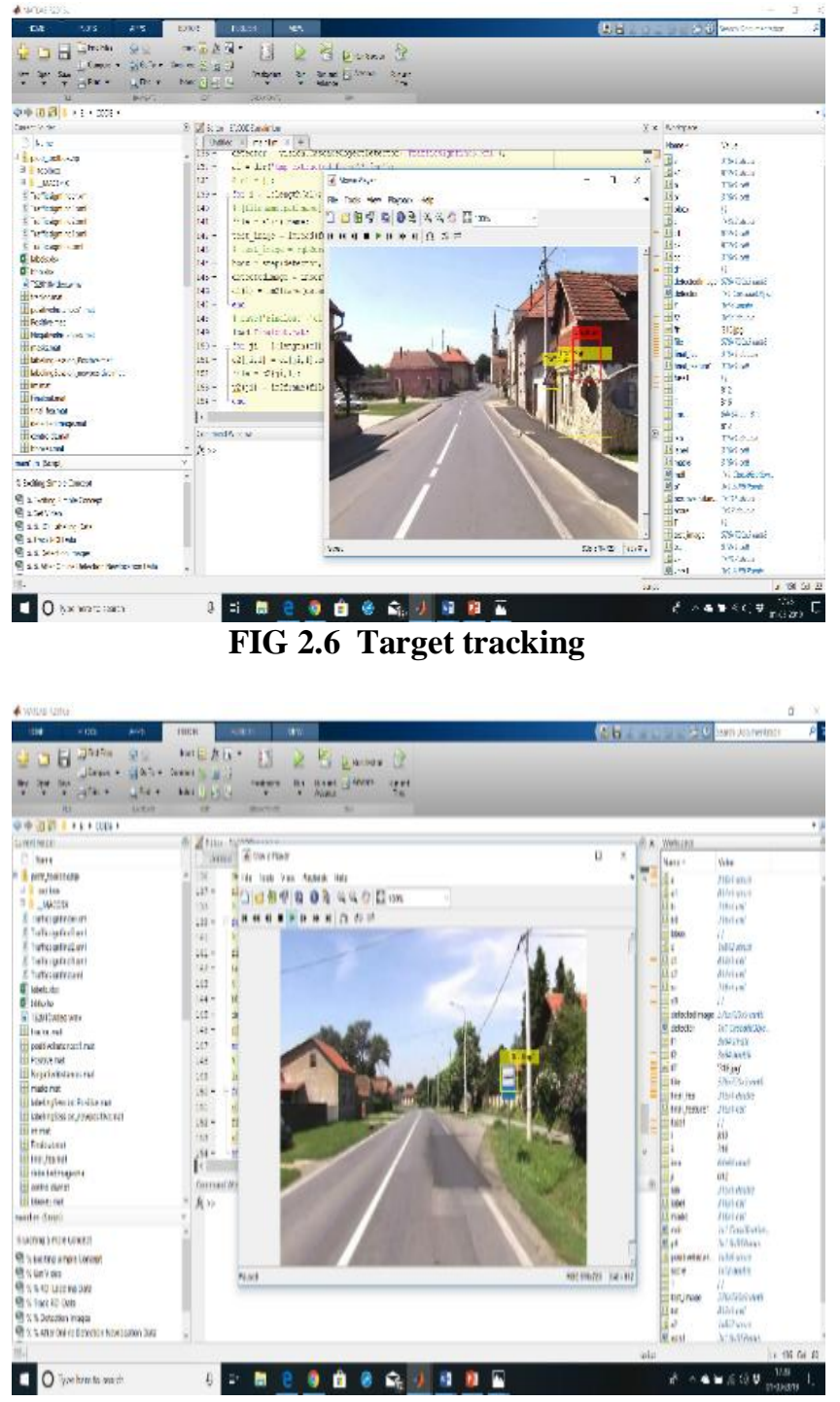

FIG 2.7 traffic sign recognition.

\section{CONCLUSION}

In this paper, a video-based traffic sign detection, following and recognition by employing a mono camera that is mounted on the vehicle is enforced. Kalmann filter was used for following the motion of the vehicle and $\mathrm{K}$ nearest algorithmic rule that provides the great performance is employed for the sign recognition. the most feature of this methodology is that it uses 2 detectors: on-line detector and offline detector, if the offline detector fails to update the knowledge, on-line detector will be used. Finally, a scale primarily based intra frame fusion technique was wont toget the results with dependants.

\section{REFERENCES}

1. "Road Traffic Sign Detection and Classification" by Arturo de la Escalera, Member, IEEE, Luis E. Moreno, Member, IEEE, Miguel Angel Salichs, Member, IEEE, and Jose Maria Armingol.

2. "Circuar Traffic Signs Recognition Using The Number Of Peaks Algorithms" by Khaled M. Almustafa, prince sultan university.

3. "An Automatic Traffic Sign Detection and Recognition System Based on Colour Segmentation, Shape Matching, and SVM" by SafatB.Wali, Mahammad A.Hannan, Aini Hussain, and Salina A.Samad.

4. "Real-Time Detection and Recognition of Road Traffic Signs using MSER and Random Forests" by XianyanKuang, Wenbin Fu, Liu Yang.

5. "Video Based Traffic Sign Detection by Scale Based Frame Fusion Technique" by Mr.K.Arun1, R.Vasanthabalan, S.Siranjeevi. vol-5,issue-3,2018.

6. "A New Approach for In-Vehicle Camera Traffic Sign Detection and Recognition" by Andrzej Ruta FatihPorikli, Yongmin Li, Shintaro Watanabe, Hiroshi Kage, Kazuhiko Sumi,2009.

7. "Road Traffic Sign Detection and Classification" by Arturo de la Escalera, Member, IEEE, Luis E. Moreno, Member, IEEE, Miguel Angel Salichs, Member, IEEE, and Jos'eMar'iaArmingol, vol-45,No.6,1997

8. "Traffic sign detection and analysis" by Ms. Yamini Gowardhan, Dr.Ramchvand Hablani ,vol-3,no-2,2016.

9. "Improved Traffic Signal Detection and Classification via Image Processing Algorithms" byLeonardo Brunoa, Giuseppe Parlaa, Clara Celauroa ,2012.

10. "Traffic signs recognition for driving assistance" by Yatham Sai Sangram Reddy1, Devareddy Karthik1, Nikunj Rana1 M Jasmine Pemeena Priyadarsini1, G K Rajini2 and Shaik Naseera3, 2017.

11. https://www.google.com/search?q=traffic+signs+pictures $\& r l z=1 C 1 C H B D+e n I N 812 I N 812 \&$ source $=\operatorname{lnms} \&$ tbm $=$ is ch\&sa $=$ X\&ved $=0$ ahUKEwjz-rCBv-

HgAhUZWX0KHY0UCygQ_AUIDigB\&biw=1366\&bih $=608$. 